\title{
Pediatric bladder/prostate rhabdomyosarcoma: Eight cases from a single center
}

\author{
Suna Emir ${ }^{1}$, Sonay İncesoy Özdemir ${ }^{2}$, Hacı Ahmet Demir ${ }^{1}$, Derya Özyörük ${ }^{1}$, \\ Esra Karakuş ${ }^{3}$, Tuğrul Tiryaki ${ }^{4}$, Faik Çetindağ 5 \\ Departments of ${ }^{1}$ Pediatric Oncology, ${ }^{3}$ Pathology, and ${ }^{4}$ Pediatric Surgery, Ankara Children's Hematology Oncology Training \\ and Research Hospital, ${ }^{2}$ Department of Pediatric Oncology, Yildırım Beyazıt University Faculty of Medicine; ${ }^{5}$ Department \\ of Radiation Oncology, Ankara Atatürk Training and Research Hospital, Ankara, Turkey. E-mail: sincesoy@yahoo.co.uk \\ Received:16 April 2016, Revised:4 August 2016, Accepted: 16 August 2016
}

SUMMARY: Emir S, İncesoy-Özdemir S, Demir HA, Özyörük D, Karakuş E, Tiryaki T, Çetindağ F. Pediatric bladder/prostate rhabdomyosarcoma: eight cases from a single center. Turk J Pediatr 2016; 58: 254-258.

In this study, clinical characteristics, treatment modalities and outcome of patients diagnosed with bladder/prostate rhabdomyosarcoma (BP RMS) were evaluated retrospectively. Files of 8 children diagnosed with BP RMS and treated between 2004-2014 were reviewed for clinical characteristics, treatment modalities and outcome. Seven males and one female were diagnosed with BP RMS between 2004-2014. Median age was 33.5 months (range, 2 to 176 months). At presentation the main clinical symptoms were hematuria in 5 patients, and constipation, oliguria and prolonged jaundice in 1 patient each. All patients were non-metastatic and only one had an embryonal histology. Primary resection before chemotherapy was performed on only one patient. Six patients were treated initially with VAC chemotherapy for 12 weeks, two patients were treated PIAV (ifosfamide, cisplatin, doxorubicin, vincristine). Local relapse or progressive disease occurred in 5 of 8 patients, and two of these patients underwent primary or secondary tumor resection without radiotherapy. Three patients developed a local relapse after combination of radiochemotherapy and tumor resection. Radical surgical treatment was performed in 3 patients with local relapse. Only one patient underwent partial cystectomy. Six of 8 patients were alive and under follow-up without disease at a median survival of 53 months (range, 13 to 78 months). BP RMS requires a multidisciplinary treatment approach. There is a general consensus that chemotherapy is the mainstay of treatment in BP RMS, but the method to be used for local control is controversial, and may vary from case to case in this heterogeneous disease.

Key words: bladder, prostate, rhabdomyosarcoma, treatment, children.

Rhabdomyosarcoma (RMS) accounts for $5 \%$ of all pediatric cancers and is the most common pediatric soft tissue sarcoma. Approximately $13-20 \%$ of all RMS cases are located in the genitourinary tract, most commonly in the bladder and prostate (BP) ${ }^{1,2}$. There is a bimodal age distribution with a peak incidence in the first 2 years of life and another in adolescence. Etiology is unknown. The tumour originates mainly in the submucosa of posterior bladder wall with particular preference for the regions of bladder neck and trigone. The main histological subtypes are embryonal and alveolar. The embryonal subtype including the botryoid variant is the most prevalent form in BP RMS. Embryonic RMS generally presents a favorable prognosis. Alveolar histology, which is characterized by two genetic reciprocal translocations PAX3-FOXO1 and PAX7-FOXO1, is more prevalent in adolescents and confers a lesser chance due to the high rate of local recurrence ${ }^{3}$. Cardinal symptoms are obstructive voiding symptoms, constipation, hematuria and abdominal mass. The vast majority of patients have disease that is localized (non metastatic) but unresectable at the time of diagnosis. The outcome of children with BP RMS has improved dramatically over the past 40 years mainly due 
to collaborative trials. The treatment regime comprises multidisciplinary and multimodal therapies including chemotherapy, radiotherapy and surgery. To date, it remains unclear which treatment strategy is optimal for locoregional control in BP RMS. Some authors advocate chemotherapy (CT) and radiotherapy (RT) followed by organ sparing surgery. Others prefer the more aggressive approach of radical surgery in patients with only partial or minimal response to chemotherapy ${ }^{4-6}$.

In this study, clinical characteristics, treatment types, and outcomes of patients with BP RMS were retrospectively evaluated.

\section{Material and Methods}

Medical records of 8 patients with BP RMS diagnosed between 2004-2014 were retrospectively evaluated. Demographic characteristics, signs and symptoms, radiological features, diagnostic procedures, pathological data, treatment modalities and outcomes of the patients were noted. Staging was performed according to the TNM pretreatment staging system on the basis of characteristics of the tumor, involvement of regional nodes and presence of distant metastases. After the initial surgery, the patients were grouped according to the postsurgical Intergroup Rhabdomyosarcoma Staging (IRS) system. IRS group I included completely resected tumors, group II included grossly resected tumors with microscopic residual disease and/or resected regional lymph node involvement, group III included gross residual tumors after incomplete resection or biopsy, and group IV included patients with metastasis at onset.

Follow-up time was considered from the diagnosis to November 2014 or last date of outpatient visit. Clinical characteristics, treatments modalities, outcomes and survival times of the patients were presented in Table I.

\section{Results}

Between 2004 and 2014, a total of 8 patients with RMS of bladder (n: 6; 1 female, 5 males) and prostate (n: 2 males) were treated at our department. Age at the time of diagnosis was between 2 and 176 months. The median age was 33.5 months. The median period of follow-up was 53 months (range: 13 to 78 months). At presentation the main clinical symptoms were hematuria in 5 patients, constipation, oliguria and prolonged jaundice in 1 patient each. According to the TNM pretreatment staging system, 3 patients had stage II RMS, 5 stage III. Seven patients underwent initial tumor biopsy (cystoscopy: 6 , tru-cut biopsy of the prostate: 1 ). Only one patient underwent primary tumor resection. According to the IRS group classification, 1 patient was classified as group II, seven patients were classified as group III. The histology was embryonal in seven and alveolar in one. Primary resection before CT was performed for only one patient and preoperative tumor rupture occurred during surgery. Seven patients received neoadjuvant CT. Five patients received treatment with VAC (vincristine, actinomycin $\mathrm{D}$ and cyclophosphamide), 2 patients received treatment with PIAV (ifosfamide, cisplatin, doxorubicin, vincristine). Assessing CT response (with computed tomography or magnetic resonance imaging and cystoscopy) was performed at 12 weeks.

Seven patients showed complete or partial responses and 1 patient did not respond to treatment. Second look surgery after neoadjuvant CT was performed in 7 patients. Vital tumor tissue was detected in 4 patients and RT was given to these patients for local control. The remaining four patients were treated with CT without RT.

Local relapse or progressive disease occurred in 5 of 8 patients. One of these underwent primary and the other one underwent secondary tumor resection without RT. Three of the patients developed a local relapse after combination of radiochemotherapy and tumor resection. There was no distant relapse. Two of the 5 patients who experienced local relapse died of progressive disease at 55 and 43 months after diagnosis, respectively. Three of the patients who experienced local relapse achieved complete remission with salvage therapy, including CT, RT and radical surgery. Details of the treatment modalities are presented in Table I.

Six of 8 patients are in remission and under follow-up without disease at a median survival of 51 months (range, 13 to 78 months). In our group of long-term survivors we detected that chemotherapy induced tubulopathy. Two 


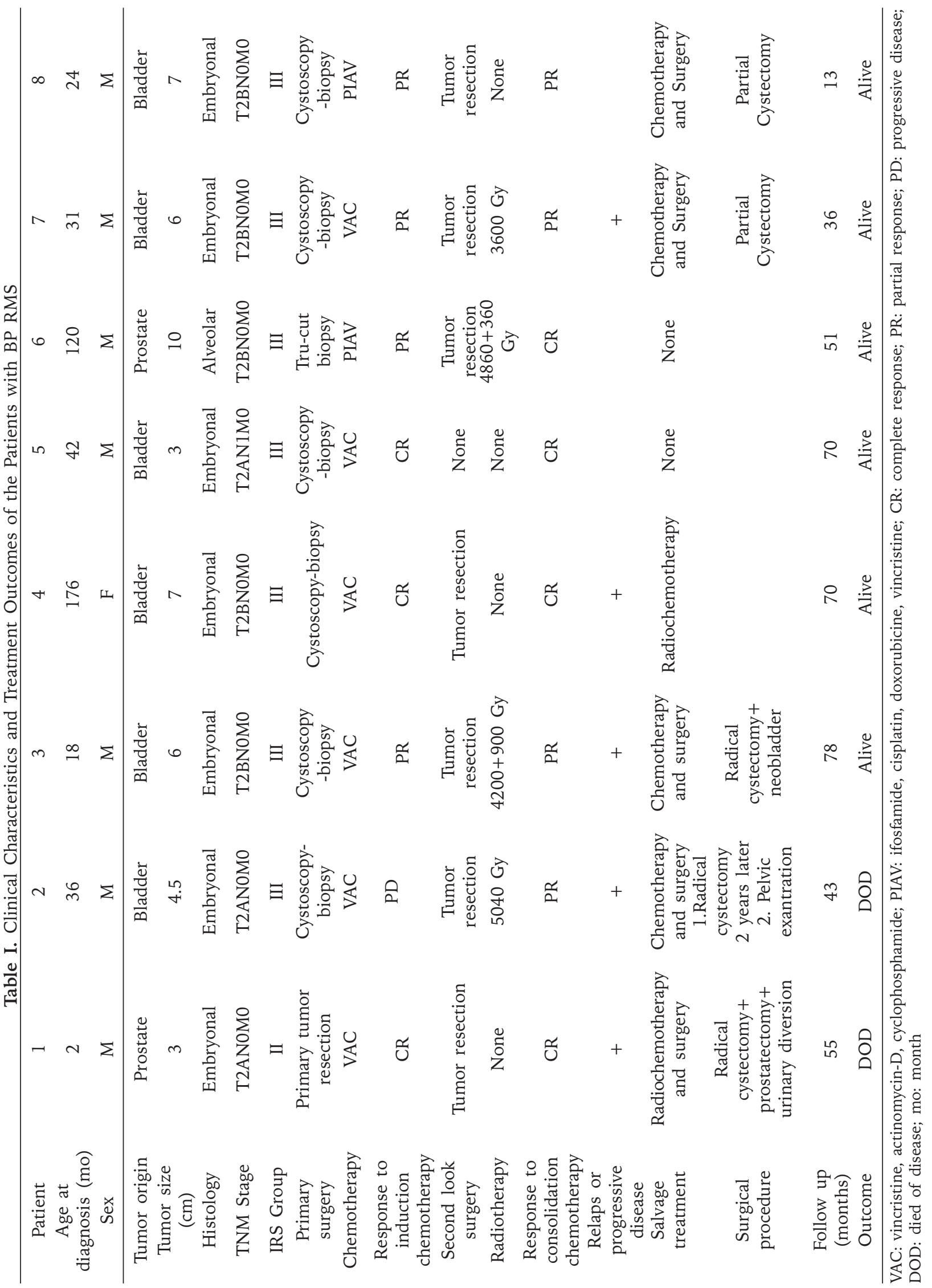


of 6 patients had mild tubulopathy. One patient (patient 3) developed severe chronic tubular toxicity. Additionally, 5 of the 6 surviving patients were continent. Patient 3, who underwent radical cystectomy-neobladder, required clean intermittent catheterization. None of the patients reviewed in this study were available for evaluation of sexual function or fertility.

\section{Discussion}

Over the past 40 years, treatment of BP RMS underwent significant changes. The use of CT for systemic control of BP RMS is well established. However, optimal treatment strategy for local control stil remains unclear, and may vary from case to case in this heterogeneous disease. Today, less aggressive surgical treatment regimens focusing on bladder preservation have been established ${ }^{7}$. Rates of cystectomy decreased from $78 \%$ in IRS-I to $9 \%$ in IRS-IV ${ }^{8}$. On the other hand, some authors advocate the significant role of radical surgery on the local control. Filipas et al. ${ }^{9}$ reported excellent outcomes with radical surgery, stressing the value of complete surgical clearance. There are no well-defined guidelines for relapsed patients. Raney et al. ${ }^{10}$ and Hayes Jordan et al. ${ }^{11}$ reported that aggressive surgery of recurrent tumor was associated with a better outcome. In our study, local relapse or progressive disease occurred in 5 of 8 patients. One of these underwent primary and the other one underwent secondary tumor resection without RT. Three of the patients developed a local relapse after combination of radiochemotherapy and tumor resection. Radical surgical treatment was performed in 3 patients with local relaps. Only one patient who had local relapse underwent partial cystectomy. Unfortunately, some patients are definitely not suitable for organ-sparing surgery, even after $\mathrm{CT}$ and RT. In cases of local relapse, radical surgical approach with cystectomy is also necessary in cases when the tumor has shown poor response to $\mathrm{CT}$ and $\mathrm{RT}^{12}$.

For the treatment of BP RMS, RT is also an option for residual tumors. However, timing and dose of radiation therapy are controversial, largely due to its added impact of local control against its late side effects. While radiotherapy reduces the number of patients who require radical excision, the lowest effective doses delivered to the bladder neck may still cause urinary incontinence, infertility, bowel injury, and bony pelvis deformity. It is unclear if these side effects cause more morbidity than surgical excision of the bladder or prostate ${ }^{12-15}$. Radiation was used as a part of initial multimodal therapy in 4 of the 8 patients (patient 2, 3, 6, 7). In patients 1 and 4 , RT was used for recurrent tumors.

In our series, 2 patients died of progressive disease at 55 and 43 months from the start of treatment. A 2-month-old patient (patient 1) with tumor of prostatic origin underwent primary tumor resection and peroperative tumor rupture occurred during surgery. After resection of the tumor, VAC protocol was given. The tumor was evaluated as complete response. The patient remained disease free lyear but the tumor relapsed. The recurrent tumor was surgically removed, and was treated with CT (cisplatin, vincristine, and adriamycin) and RT. This patient experienced a second local relapse, which again was treated with surgery (radical cystoprostatectomy with urinary diversion) and CT. Unfortunately, after more intensive $\mathrm{CT}$, the patient died because of progressive disease. Patient 2 had IRS group III disease and no response to induction CT. Tumor was surgically removed, and was treated by more intensive CT and RT (5040 cGy). Because of progressive disease, the patient underwent radical surgery. But, the patient died at 43 months after the diagnosis.

Our study has some limitations because of the small number of the study group. Besides, our study included a heterogeneous group of patients in the cohort, and therefore it is difficult to draw a conclusion of which approach truly contributes to the survival of the patients better. These data would be informative to optimize the treatment balance for children with BP RMS.

In conclusion, BP RMS requires a multidisciplinary treatment approach. Because of various complications and morbidity caused by different approaches, optimal outcome is dependent on close collaboration between surgical, radiotherapy, and pediatric oncology specialists ${ }^{16}$. Also, further prospective studies in children with BP RMS are needed in this field. 


\section{REFERENCES}

1. Stein R, Frees S, Schröder A, et al. Radical surgery and different types of urinary diversion in patients with rhabdomyosarcoma of bladder or prostate--a single institution experience. J Pediatr Urol 2013; 9: 932-939.

2. Hishiki T, Saito T, Mitsunaga T, et al. Optimal surgical treatment and urological outcomes in boys with pelvic and urogenital rhabdomyosarcomas and soft tissue sarcomas. Pediatr Surg Int 2013; 29: 1077-1082.

3. Denes FT, Duarte RJ, Cristofani LM, Lopes RI. Pediatric genitourinary oncology. Front Pediatr 2013; 1: 48.

4. Seitz G, Dantonello TM, Int-Veen C, et al. Treatment efficiency, outcome and surgical treatment problems in patients suffering from localized embryonal bladder/prostate rhabdomyosarcoma: a report from the Cooperative Soft Tissue Sarcoma trial CWS-96. Pediatr Blood Cancer 2011; 56: 718-724.

5. Jenney M, Oberlin O, Audry G, et al. Conservative approach in localised rhabdomyosarcoma of the bladder and prostate: results from International Society of Paediatric Oncology (SIOP) studies: malignant mesenchymal tumour (MMT) 84, 89 and 95. Pediatr Blood Cancer 2014; 61: 217-222.

6. Alexander N, Lane S, Hitchcock R. What is the evidence for radical surgery in the management of localized embryonalbladder/prostate rhabdomyosarcoma?Pediatr Blood Cancer 2012; 58: 833-835.

7. Komasara L, Golebiewski A, Anzelewicz S, Czauderna P. A review on surgical techniques and organ sparing procedures in bladder/prostate rhabdomyosarcoma. Eur J Pediatr Surg 2014; 24: 467-473.

8. Arndt C, Rodeberg D, Breitfeld PP, Raney RB, Ullrich F, Donaldson S. Does bladder preservation (as a surgical principle) lead to retaining bladder function in bladder/prostate rhabdomyosarcoma? Results from intergroup rhabdomyosarcoma study iv. J Urol 2004; 171: 2396-2403.
9. Filipas D, Fisch M, Stein R, Gutjahr P, Hohenfellner $\mathrm{R}$, Thüroff JW. Rhabdomyosarcoma of the bladder, prostate or vagina: the role of surgery. BJU Int 2004; 93: $125-129$

10. Raney RB Jr, Crist WM, Maurer HM, Foulkes MA Prognosis of children with soft tissue sarcoma who relapse after achieving a complete response. A report from the Intergroup Rhabdomyosarcoma Study I. Cancer 1983; 52: 44-50.

11. Hayes Jordan A, Doherty DK, West SD, et al. Outcome after surgical resection of recurrent rhabdomyosarcoma. J Pediatr Surg 2006; 41: 633-638.

12. Wu HY, Snyder HM 3rd, Womer RB. Genitourinary rhabdomyosarcoma: which treatment, how much, and when? J Pediatr Urol 2009; 5: 501-506.

13. Cecchetto G, Carretto E, Bisogno G, et al. Complete second look operation and radiotherapy in locally advanced non-alveolar rhabdomyosarcoma in children: A report from the AIEOP soft tissue sarcoma committee. Pediatr Blood Cancer 2008; 51: 593-597.

14. Kuru TH, Roethke M, Fenchel M, Hadaschik BA. How could imaging reduce therapy-associated morbidity in rhabdomyosarcoma of the bladder or prostate? Future Oncol 2013; 9: 1797-1800.

15. Rodeberg DA, Anderson JR, Amdt CA, et al Comparison of outcomes based on treatment algorithms for rhabdomyosarcoma of the bladder/prostate: combined results from the Chidren's Oncology Group, German Cooperative Soft Tissue Sarcoma Study, Italian Cooperative Group and International Society of Pediatric Oncology Malignant Mesenchymal Tumors Committee. Int J Cancer 2011;128: 1232-1239.

16. Ferrer FA, Isakoff M, Koyle MA. Bladder/prostate rhabdomyosarcoma: past, presentand future. J Urol 2006; 176: 1283-1291. 